\title{
Commentary \\ Benefits of Anti-Aging Actives in Sunscreens
}

\author{
Karl Lintner
}

KAL'IDEES S.A.S., Paris 75014, France; karl.lintner@kalidees.com

Academic Editors: Johanna Maria Gillbro and Enzo Berardesca

Received: 28 October 2016; Accepted: 19 January 2017; Published: 25 January 2017

\begin{abstract}
Sunscreens are functional, utilitarian, cosmetic products. The criteria of purchase are different from those for skin care and make-up. Companies are trying to add glamour and value to basic sunscreens by incorporating "active" ingredients (other than UV filters) into these formulas and by communicating about the additional benefits, be they anti-aging, moisturizing, firming, anti-wrinkle, etc. While some of these ideas of additional ingredients make sense as supplementary skin protection, some others do not afford much benefit in view of the infrequent application and short period of usage. The present article reviews some of these ideas and presents a few active ingredients that might be of value in such a context, even if substantiation of such additional claims in sunscreens is often lacking.
\end{abstract}

Keywords: anti-age; vitamins; free radicals; skin protection; infrared radiation

\section{Introduction}

Sunscreens have come a long way since the first "tanning oils" were marketed and the first UV filters were incorporated. Today, sunscreens are sophisticated formulas that promise and deliver more than just UV blockage and the avoidance of sunburn. As in so many other domains, we see a blurring of frontiers, a mixing of categories, a (perhaps deliberate) gradual disappearance of clear distinctions and definitions.

The term "sunscreen" suggests to most consumers the general category of cosmetic products used on the skin during extensive sun exposure in order to protect the skin against the deleterious effects of direct sunlight, although sometimes the word is also understood to mean the specific UV filters and reflectors. We shall retain the general terminology of "sunscreens" as the finished cosmetic consumer products that bear a clear message of "protection against solar radiation", such as the prevention of erythema, sunburns, and sometimes even cancer. This would include, in most cases, "sun tan lotions", "sun care products", and "sun-blocks", but would exclude "after-sun lotions" and "self-tanning products".

The difficulties in nomenclature arise also because of different legislation in different parts of the world and because of technical and marketing considerations; a "sunscreen" of today contains, more and more often, specific skin and/or body care active ingredients, accompanied by corresponding claims, which are the subject of this review paper. On the other hand, an increasing number of classical "skin care" (i.e., face care, lip care, make-up, or body-care) products boast Sun Protection Factors (SPFs) in the 5-30 range. These products have primary skin care claims (moisturizing, anti-wrinkle, firming, etc.) and offer the sun protection as an additional benefit. So where is the borderline between the two? A "sunscreen" of SPF30 with an additional anti-age claim is seen as a "sunscreen" (for example, Clinique's Body Cream SPF30 with antioxidants) because the marketer positions the product as such (design of tube and packaging, advertising, point of sale, and timing of promotional activity), whereas Clinique's Superdefense Daily Defense Moisturizer has an of SPF20 but is clearly NOT positioned as a "sunscreen". It also claims to contain antioxidants and is a "classical", albeit innovative, face care product. The difference between the two products? The feel and texture of the products against the 
skin and the price. Neutrogena's "Healthy Skin Anti-Wrinkle Intensive Deep Wrinkle Moisturizing Treatment SPF 20" also seems to add the SPF claim as an afterthought.

How about "Actives"? Except for the categories called Over the Counter or OTC) (in the USA), Quasidrug (in Japan), and Functional Cosmetics (in Korea) in the respective countries, the notion of a cosmetic "Active" has no legal basis, contrary to pharmaceutical "Actives" [1]. How then to define the term in the cosmetics (i.e., personal care) domain? Briefly, any cosmetic ingredient that has (i) individually demonstrated reasonable specific and more pronounced cosmetic activity on human skin (or its appendages) compared to a vehicle, (ii) a well substantiated claim, and (iii) a plausible "story" with a mechanism of action to go with it can be considered an "Active". This encompasses, then, the wide field of ingredients (such as are found in the Personal Care Product Council (PCPC) repertoire or in the dictionary called International Nomenclature of Cosmetic Ingredients (INCI) dictionary) from botanicals (various types of plant extracts) to pure chemical entities that possess a function that is clearly different from their galenic purpose (emulsifiers, texturizers, thickeners, preservatives, fragrances, etc.). Again, it may occur that an ingredient functions as an "Active" in one product (positioned as such by the marketer) and as a basic ingredient in another product (glycerine, lecithin, and lanolin come to mind). In general, an ingredient becomes an "Active" when its marketers decide to position it as such, ignoring the cosmetic "activity" of the other ingredients. In this article, we shall concentrate on "Actives" that are particularly suited and/or already in use in the general category of "sunscreens"; "Actives" that make sense in the context of sun care and skin protection/treatment.

We can therefore now drop the quotation marks from the word Active.

Finally, "anti-aging" (again with quotation marks): the term is catchy, seductive, but very vague. Some countries (e.g., Canada) have regulations forbidding the use of this "claim" in cosmetic advertising or on the packaging.

As usual, there are two aspects to the concept, prevention and treatment. Anti-Age Prevention implies that a consumer product helps "reduce the speed of the appearance of the clinical signs of (cutaneous) aging", based on the protective Active contained in the product. Anti-Age Treatment promises to reverse (some of) the visible signs of skin aging (through activities such as wrinkle reduction, firmness improvement, moisturisation of dry skin, etc.), based on Actives that "restore, regenerate, repair ... " skin items such as "barrier, extracellular matrix, collagen fibers, and hydrolipid balance" alongside similar parameters.

To treat the entire subject of Anti-ageing (which of course must start with the study of the mechanisms of ageing) is beyond the scope of this paper.

In the following we shall concentrate on cosmetic realities and consider both types of "Anti-aging" actives (prevention and treatment) and their respective merits in sunscreens. However, if modern UV filters are so well suited to protect us against the sun's dangerous rays, for what reason (other than a marketing and/or label claim) should the formulator of a modern sunscreen add anti-aging Actives to his product?

For one, and most importantly, UV filters are not absolute; even an SPF60 (not everywhere allowed as a claim) will wear off with time or may not be applied in sufficient amounts from the beginning; or the sun exposure of the person wearing it continues beyond the period of protection [2,3]. Any well chosen additional Active in the product will help to decrease the damages that are not prevented by the UV filter. For instance, this seems to be particularly true for the combination of UV-A filters with anti-oxidant protective molecules. The presently available UV-A filters are rarely able to block out all of the UV-A radiation so as to prevent all free radical generation in the deeper layers of the skin [4] and references therein.

Secondly, we observe a trend in all cosmetic formulas and products, wherein make-up mascaras, foundations, lip sticks, powders, cleansers, and body care and scalp care SKUs (Stock Keeping Units) all contain Actives for additional benefits. True skin care needs a global and continuous approach. We need the sunlight for the synthesis of Vitamin D and for our psychic well being, and we desire silky youthful skin; for this we need the optimum combination of sunscreen and skin care Actives. 
As Dr. Rosengarten (Laboratoire Dermatologique La Roche Posay S.A.S., La Roche Posay, France) said, "we see it as our responsibility not to rely on fear, but instead to use pampering to encourage people to use more extensive protection against UV rays" [5].

Interestingly, these combination products cover a wide range, which includes moisturizers with an SPF20 or more, anti-wrinkle products with high sun-protection, and firming claims side by side with SPF. Furthermore, we also find a skin whitening product with SPF50 on one hand and a tanning lotion with SPF50 on the other hand.

Hence the question: what Actives does it make sense to add to a sunscreen? Preventative or curative ingredients?

\section{Strategies of Anti-Aging Actives in Sunscreens}

\subsection{Prevention of Damages ("Slowing Down the Aging Process")}

\subsubsection{Vitamins}

One of the first and most widely used categories of Actives formulated in sunscreens is the one comprising the vitamins $\mathrm{C}$ and $\mathrm{E}$, sometimes $\mathrm{A}$ (retinoids) or a few of the B group.

The literature on the effects of vitamins $\mathrm{C}$ and $\mathrm{E}$ as photoprotective agents in cell cultures (in vitro) and on animals is impressively large. Although general consensus is expressed that the protective effect afforded by these molecules "might be beneficial" to human skin, there is astonishingly little documentation of the benefits of vitamin uses in cosmetic finished products, especially in sunscreens, on human skin (in vivo). Thiele et al. [6] in 2005 said: "The lipophilic antioxidant vitamin E has been used for more than 50 years in clinical and experimental dermatology. However, although a large number of case reports were published, there is still a lack of controlled clinical studies providing a rationale for clinical indications and dosage."

It is not the purpose of this paper to present an exhaustive review of this topic, as a rapid overview of the literature shows that research into the effects of topical vitamin application continues, with many papers focusing on combinations of vitamins, such as E plus C or E plus A.

Only a few clinical trials on humans seem to have been carried out over the last decade. Burke [7] reviews studies of combinations of vitamins $\mathrm{C}$ and $\mathrm{E}$, concluding that the synergy in UV protection arises from the mutual regeneration of the vitamins. Murray et al. [8] and Oresajo et al. [9] in a follow up study to Lin [10] (all publications from Pinnell's laboratory) show the in vivo protective effect of stabilized topical antioxidant blends containing vitamin C, ferulic acid, and either phloretin or tocopherol.

Sivapirabu et al. [11] report that topical nicotinamide provides some protection against UV-induced immunesuppression.

In summary, "vitamins are good for you". Their use in sunscreens is widespread; based on numerous studies, even if most of them are animal or in vitro studies, their claim to "anti-aging" activity is not far-fetched. Vitamins C and E would be considered more of the "preventative" type (anti-oxidant, to be used before or during sun exposure), while vitamin A and its derivatives are more the "repair" type, undoing some of the damages caused by US. Their main drawback is the difficulty in formulating stable vehicles, such that the right amount of efficacious vitamins can be guaranteed for sufficient shelf life.

\subsubsection{Botanicals}

Botanicals, also known as "plant extracts", merit a short mention here, as an increasing number of ingredients of plant origin are offered and used that are tested and positioned as "anti-aging" actives and are thus used or useful in sunscreens.

Plant extracts cover the spectrum from hydroglycolic solutions of an analytically ill defined nature to pure, isolated, chemically identified molecules and include all products that present intermediate stages of purification. The reputation of the plant kingdom is one of an almost unlimited source of potential healing activities; with thousands of substances yet undiscovered. 
A closer look reveals, however, that, with some notable exceptions, a few broad categories suffice to describe the benefits obtained from plant extracts for cosmetic claims; we find antioxidant activity (polyphenols, vitamins, and flavonoids), anti-inflammatory properties (many of them being non-steroidal enzyme inhibitors such as ursolic acid, oleanolic acid, nordihydroguaiaretic acid (NDGA), and others), and tissue repair molecules (di- and triterpenes). All of these activities can be employed for "anti-age" claims; all of them make some sense in the context of sunscreen formulation. Green tea is a favourite among the botanicals with a well-known reputation. In vitro scavenging of hydrogen peroxide and the prevention of UV induced oxidative damages on skin cells in culture by various fractions of green and black teas, including purified epigallocatechin gallate (EGCG), were described by Wei et al. [12].

Care must however be taken in dosing these ingredients so as to avoid vasodilatation and skin redness, rashes, and allergy. Furthermore, as plants are naturally designed to absorb sunlight (for photosynthesis), their extracts usually contain ingredients that show photo absorption, thus requiring specific phototoxicity studies.

A case in point is shown in the paper by Martin et al. [13] from the Johnson \& Johnson company; these authors describe the benefits of a Feverfew (Tanacetum parthenium) extract which possesses free radical scavenging activity and reduced UV generated erythema, epidermal hyperplasia, DNA damage, and apoptosis. However, the authors took care to obtain a parthenolide-depleted extract, as this sesquiterpene lactone is reputed to be allergenic, although it is the principal active ingredient for most other applications of this botanical medicine.

Reviews of photo-chemoprevention by botanical anti-oxidants, in view of their use in sunscreens, are given in [14] and in a recent meta-analysis by Saric and Sivamani [15]. This latter article nevertheless demonstrates the scarcity of vehicle controlled human trials on well-defined extracts.

\subsubsection{Enzymes}

The idea of using antioxidant enzymes to protect the skin has received less attention in the sunscreen and protection fields. There are two reasons that may account for this; technical difficulties in analyzing enzyme activities on human skin and the inherent instability of enzymes, which makes them hard to formulate and stabilize in finished cosmetic sunscreens. Nevertheless, basic research into the innate enzyme defense system of the skin has progressed, and a number of in vitro, animal, and human in vivo studies point to the delicate balance that is required between the various enzymes in the skin.

The molecules present in the skin that are supposed to protect us against these damages are the vitamins (see above), a few other antioxidants (melanin, urocanic acid, glutathione, and ubiquinone) and specific enzymes, essentially superoxide dismutase (SOD), glutathion peroxidase (GPO) and catalase.

It now has become evident that these inherent antioxidant defense systems of the skin are rapidly overwhelmed by the amount of sun exposure with which we stress them through contemporary life styles. Not only vitamins $\mathrm{C}$ and $\mathrm{E}$ are depleted in the skin by UV irradiation; the same occurs with the enzymes. Miyachi et al. [16] describe the decrease of SOD activity after a single dose of UV light; Pence and Naylor [17] confirm this observation in hairless mice and add that catalase activity also was significantly depressed. Punnonen et al. [18] extended this observation to human epidermis. A quantitative analysis of the localization of these enzymes (and non-enzymatic antioxidants) in murine skin and their decrease after UV exposure is presented by Shindo et al. [19]. A thorough investigation on humans, carried out over winter and summer seasons, confirms this fact; catalase is easily destroyed by UV-A light in summer and more active in winter; whereas SOD is much more resilient [20]. This then leads to a potential build-up of hydrogen peroxide in the skin, which is not necessarily the best thing to occur. The need for a balanced anti-oxidant enzyme system thus becomes apparent.

Two approaches are possible: (a) to stimulate and/or protect the innate enzyme system so that, even under UV exposure, it retains its efficacy or (b) to supply the lacking enzymes by topical application, for instance within a sunscreen, as well as pre-sun or post-sun products. 
Udo Hoppe and his colleagues [21], as well as Daniel Maes and his co-workers [22], presented examples of the first strategy; they show that molecules such as salicin in skin fibroblasts (Hoppe) and vitamin D derivatives or betulinic acid in keratinocytes (Maes) are able to stimulate the synthesis of heat shock proteins, which protect the catalase against UV induced degradation. These molecules could therefore be used advantageously in sunscreens as anti-aging Actives inasmuch as they induce protection of our own anti-aging defense systems. The extract of Sophora japonica flowers (RESISTRESS ${ }^{\circledR}$ of Solabia S.A.S., Pantin, France) was enriched in engineered quercetin dimers and has been shown to stimulate the synthesis of the skin's own SOD and catalase enzymes.

Strategy (b) has a few limitations. Usually available enzymes such as SOD and catalase (extracted from animal tissue, yeast, or other biotech sources) are not easy to stabilize in cosmetic formulas, to say the least. Complicated packaging stratagems or encapsulation techniques may overcome the problem; it is however well known that enzymes, relatively large proteins, are inherently unstable in aqueous environments and also heat and UV sensitive. Furthermore, SOD alone on the skin would lead, at least theoretically, to a build-up of hydrogen peroxide, already decried by Maes [20] as being the "natural" problem of the seasonal variations of these enzyme activities. Adding the fragile catalase is not only difficult, it is also not possible for any formula sold in Europe because of an archaic prohibition of catalase use in cosmetic products [23].

A neat solution to this problem is afforded by anti-oxidant enzymes originating from organisms that live and thrive under extreme conditions of heat: the "extremophiles". Discovered a bit more than a decade ago, these bacteria live close to the hydrothermal vents at the bottom of the ocean, at temperatures that can reach $80-100{ }^{\circ} \mathrm{C}$. An active ingredient based on this concept is described by Mas Chamberlin et al. [24,25]. Thermus thermophilus bacteria, harvested 6000 feet below the California coast, are fermented at $75{ }^{\circ} \mathrm{C}$ then extracted and concentrated to yield a high potency solution containing enzyme activity that mimics superoxide anion dismutating (SOD), hydrogen peroxide converting, and glutathion peroxidase-like properties. Results of in vitro tests carried out on this cosmetic ingredient include the protection of human fibroblasts in culture, lipoperoxidation inhibition, protection of DNA against the formation of 8-oxo-guanidine, and collagen contraction [25,26]. Studies on human volunteers show the persistence of cutaneous catalase protection against UVA irradiation and a decrease of in vivo lipoperoxidation of the stratum corneum. Recent further investigations even confirmed protection against Infrared-A induced free radicals (VENUCEANE ${ }^{\mathrm{TM}}$ of SEDERMA S.A.S., Le Perray en Yvelines, France) [27].

The general public has by now understood the need for sun protection but equates this need with trips to the beach or the ski slopes, forgetting that UVA is a constant threat to the skin, even in a parking lot or the back yard. A more recent study on these extremozymes attempted to address this question; is it possible to demonstrate, on human volunteers, the visible and measurable benefits of daily sun protection by other means than an SPF? To this end, 50 volunteers were recruited on Mauritius Island (a tropical climate with a high UV index all year round). The important inclusion criterion for these panelists of relatively fair skin (phototype II-III) was to have only recently ( $<6$ months) arrived on the island, thus having skin not yet well adapted to the climate. They were then divided into two groups, receiving either a placebo moisturizer or one containing the above extremozyme preparation. Their skin quality, Transepidermal Water Loss (TEWL), wrinkles and other age related symptoms were measured before the start of the test and then over 1,3, and 6 months. As might be expected, the placebo group showed visible signs of ageing in the facial skin and on the forearms, whereas the group using the protective enzymes did not show this quick deterioration of their skin condition [26]. Thus, it appears that this original clinical study protocol shows, maybe for the first time, the visible effects of anti-age UVA prevention beyond the classical sun burn protection.

A different approach to UV protection using an enzyme is given by Giacomoni [28]. He indicates the use of DNA repair enzymes, encapsulated in liposomes, to accelerate the removal of the pyrimidine dimers formed after UV irradiation. Although this is a repair mechanism, it still would rate as 
protection for the skin against further graver damages, rather than as a tissue repair concept as discussed in the later part of this chapter.

\subsubsection{Miscellaneous}

A few more (non-exhaustive) ingredients of a diverse nature that might be of interest in photoprotection can be found in the technical literature.

- The Vincience division of Ashland describes a synthetic peptide (Lumikit ${ }^{\mathrm{TM}}$ biofunctional) that protects the Dinein/Dinactin melanosome transfer complex against UV-induced disturbance, thus protecting the keratinocyte nucleus by erecting a kind of umbrella, an innovative protective mechanism.

- An extract of Butterfly Bush (Buddleja officinalis), which is rich in verbascoside and echinacoside, called SOLIBERINE ${ }^{\mathrm{TM}}$ (Greentech S.A.S., Saint Beauzire, France) is shown to inhibit the trans-cis transition of urocanic acid in the skin. Urocanic acid, initially in the trans form in the skin, is changed into its cis configuration, which has been shown to be involved in UV-induced immune suppression and even linked to cancer initiation [29]. Hence the Soliberine ${ }^{\mathrm{TM}}$ potency to inhibit this change from trans to cis is of interest in a sunscreen; the active molecules of the extract also possess a UVA absorbing potency, an additional benefit.

- A very well described pure designer peptide (Acetyl-hexapeptide-6 from Lucas Meyer Cosmetics Ltd., with the trade name MELITAN ${ }^{\mathrm{TM}}$ in Canda) is claimed to act on the MSH (melanostimulating hormone) receptor of melanocytes called MCR-1 and to thus increase c-AMP synthesis, leading to more active tyrosinase enzymes and melanogenesis. Both in vitro and clinical tests confirm the hypothesis of an increased tanning rate.

\subsection{Treatment of UV Induced Age Symptoms}

As mentioned in the introduction, "reversing" some of the signs of aging is of course also considered "anti-age" activity. Is it realistic? Can anything but retinoic acid reduce some of the wrinkles, the sagging skin, the dryness, and loss of tonus that come with (photo)-aging?

The sunscreen market has already acted and has begun to introduce sunscreens that contain various actives with some type of anti-age and repair claims. Recent work in numerous laboratories has shown that, indeed, some powerful ingredients with biological activity can help restore some of the youthful appearance that has suffered over time.

It is not possible to review here the enormous mass of anti-age and wrinkle repair ingredients of synthetic, marine, botanical, or biotechnological origin proposed on the market, which all might be considered, based on their merit, for inclusion in sunscreens. Contrary to the "prevention type" products discussed above, the interaction between the repair active and the sunscreen and/or the UV irradiation is not guaranteed and most combinations of "anti-age" treatment in a sunscreen make little sense, given the short periods of application (vacation times ... ). But some dermatologists hold the opinion that, while a sunscreen will not reduce wrinkles by itself, it may be a good marketing idea to include such anti-age "repair" ingredients, as this allows the formulator to encourage people to use sunscreens on a daily basis [30].

There is at least one product proposed (JUVELEVEN ${ }^{\mathrm{TM}}$ from Lipotec, Gava, Spain) that seems to justify a repair mechanism operating in a sunscreen. In a study to this effect, the skin (inner forearm) of volunteers was UV irradiated (compared to control subjects) then treated with the peptide-containing preparation; suction blisters were used six hours later to extract fluid from the skin, in which DNA could be found and analyzed for the appearance of cyclobutane pyrimidine dimer (CPD)-dimers. It was shown that the peptide-treated previously irradiated sites contained significantly fewer CPD dimers, a clear indication that a repair process has occurred, as the peptide was not present before or during the irradiation [31].

To demonstrate the repair of UV-induced damages to DNA remains a challenge. 


\section{Conclusions}

I have tried to demonstrate that the notion of Anti-Aging Actives in sunscreens opens many possibilities to formulators for improving basic sunscreen products, to add real benefits, and to allow for a variety in claims and marketing positioning.

The prevention of sun damages to the skin can be reinforced by some of the antioxidant and photoprotective agents; the treatment of sun damages during or immediately after sun exposure with repair actives is also justified. Teaching the consumer how to "manage" the sunlight (.) has become part of the marketer's obligation; prevention goes beyond using sunscreens and includes wearing adequate clothes and avoiding the hottest hours of the day, etc.

Depending on the country, however, from the US to Europe to East Asia, the legislations on sunscreens, claims, and formulations are quite different and complex. Adding certain anti-age Actives to these sunscreens may make sense in some cases, as outlined, but it makes the legal situation even more complex with respect to advertised claims. Furthermore, except for very rare instances, none of these Actives have been tested in real sunscreen conditions against identical sunscreen vehicles (without the Actives); an exception is Grether-Beck's study on an antioxidant complex described in [32]. Careful wording of any anti-aging claims on sunscreens is thus recommended, no matter how many studies one cites in support of this added benefit.

Conflicts of Interest: The author declares no conflict of interest.

\section{References}

1. Lintner, K. The Role of Actives in Face Care. SÖFW-J. 2003, 129, 2-8.

2. Pinnell, S.R. Cutaneous photodamage, oxidative stress and topical protection. J. Am. Acad. Dermatol. 2003, 48, 1-19. [CrossRef] [PubMed]

3. Petersen, B.; Datta, P.; Philipsen, P.A.; Wulf, H.C. Sunscreen use and failures-On site observations on a sun-holiday. Photochem. Photobiolog. Sci. 2013, 12, 190-196. [CrossRef] [PubMed]

4. Pustisek, N.; Situmm, M. Review Protection against Solar Ultraviolet Radiation in Childhood. Coll. Antropol. 2011, 35, 343-346. [PubMed]

5. Rosengarten, N. Sun protection as a pampering treatment. COSSMA Mag. 2016, 2, 22.

6. Thiele, J.J.; Hsieh, S.N.; Ekanayake-Mudiyanselage, S. Vitamin E: Critical review of its current use in cosmetic and clinical dermatology. Dermatol. Surg. 2005, 31, 805-813. [CrossRef] [PubMed]

7. Burke, K.E. Interaction of vitamins C and E as better cosmeceuticals. Dermatol. Ther. 2007, 20, 314-321. [CrossRef] [PubMed]

8. Murray, J.C.; Burch, J.A.; Streilein, R.D.; Iannacchione, M.A.; Hall, R.P.; Pinnell, S.R. A topical antioxidant solution containing vitamins $\mathrm{C}$ and $\mathrm{E}$ stabilized by ferulic acid provides protection for human skin against damage caused by ultraviolet irradiation. J. Am. Acad. Dermatol. 2008, 59, 418-425. [CrossRef] [PubMed]

9. Oresajo, C.; Stephens, T.; Hino, P.D.; Law, R.M.; Yatskayer, M.; Foltis, P.; Pinnell, S.R. Protective effects of a topical antioxidant mixture containing vitamin $\mathrm{C}$, ferulic acid, and phloretin against ultraviolet-induced photodamage in human skin. J. Cosmet. Dermatol. 2008, 7, 290-297. [CrossRef] [PubMed]

10. Lin, J.Y.; Selim, M.A.; Shea, C.R.; Grichnik, J.M.; Omar, M.M.; Monteiro-Riviere, N.A.; Pinnell, S.R. UV photoprotection by combination topical antioxidants vitamin C and vitamin E. J. Am. Acad. Dermatol. 2003, 48, 866-874. [CrossRef] [PubMed]

11. Sivapirabu, G.; Yiasemides, E.; Halliday, G.M.; Park, J.; Damian, D.L. Topical nicotinamide modulates cellular energy metabolism and provides broad-spectrum protection against ultraviolet radiation-induced immunosuppression in humans. Br. J. Dermatol. 2009, 161, 1357-1364. [CrossRef] [PubMed]

12. Wei, H.; Zhang, X.; Zhao, J.F.; Wang, Z.Y.; Bickers, D.; Lebwohl, M. Scavenging of hydrogen peroxide and inhibition of ultraviolet-light induced oxidative DNA damages by aqueous extracts from green and black teas. Free Radic. Biol. Med. 1999, 26, 1427-1435. [CrossRef]

13. Martin, K.; Sur, R.; Liebel, F.; Tierney, N.; Lyte, P.; Garay, M.; Oddos, T.; Anthonavage, M.; Shapiro, S.; Southall, M. Parthenolide-depleted Feverfew (Tanacetum parthenium) protects skin from UV irradiation and external aggression. Arch. Dermatol. Res. 2008, 300, 69-80. [CrossRef] [PubMed] 
14. Afaq, F.; Mukhtar, H. Photochemoprevention by botanical antioxidants. Skin Pharmacol. Appl. Skin Physiol. 2002, 15, 297-306. [CrossRef] [PubMed]

15. Saric, S.; Sivamani, R.K. Polyphenols and Sunburn. Int. J. Mol. Sci. 2016, 17, 1521. [CrossRef] [PubMed]

16. Miyachi, Y.; Imamura, S.; Niwa, Y. Decreased skin superoxide dismutase activity by a single exposure of ultraviolet radiation is reduced by liposomal superoxide dismutase pretreatment. J. Invest. Dermatol. 1987, 89, 111-112. [CrossRef] [PubMed]

17. Pence, B.C.; Naylor, M.F. Effects of single-dose ultraviolet radiation on skin superoxide dismutase, catalase and xanthine oxidase in hairless mice. J. Invest. Dermatol. 1990, 95, 213-216. [CrossRef] [PubMed]

18. Punnonen, K.; Autio, P.; Kiistala, U.; Ahotupa, M. In Vivo effects of solar-simulated ultraviolet irradiation on antioxidant enzymes and lipid peroxidation in human epidermis. Br. J. Dermatol. 1991, 125, 18-20. [CrossRef] [PubMed]

19. Shindo, Y.; Witt, E.; Packer, L. Antioxidant defense mechanisms in murine epidermis and dermis and their responses to ultraviolet light. J. Invest. Dermatol. 1993, 100, 260-265. [CrossRef] [PubMed]

20. Hellemans, L.; Corstjens, H.; Neven, A.; Declercq, L.; Maes, D. Antioxidant enzyme activity in human stratum corneum shows seasonal variation with an age-dependent recovery. J. Invest. Dermatol. 2003, 120, 434-439. [CrossRef] [PubMed]

21. Steckel, F.; Gieseler, R.; Pollet, D.; Hoppe, U. Heat shock protein expression and UV-light induced damage in cultured human skin cells. In Proceedings of the $X X^{\text {th }}$ Congress International Federation of the Societies of Cosmetic Chemists, Cannes, France, 14-18 September 1998; pp. 133-140.

22. Declercq, L.; Hellemans, L.; Goyarts, E.; Gan, D.; Corstjens, H.; Sente, I.; Mammone, T.; Marenus, K.; Maes, D. Induction of heat shock proteins to protect against seasonal deficiency in antioxidant defense capacity of human stratum corneum. In Proceedings of the XXII ${ }^{\text {th }}$ Congress International Federation of the Societies of Cosmetic Chemists, Edinburgh, UK, 24-27 September 2002.

23. Regulation (EC) No 1223/2009 of the European Parliament and of the Council of 30 November 2009 on Cosmetic Products (Text with EEA Relevance). Available online: http://eur-lex.europa.eu/eli/reg/2009/ 1223/oj (accessed on 26 October 2016).

24. Mas-Chamberlin, C.; Lamy, F.; Mondon, P.; Scocci, S.; de Givry, L.; Vissac, F.; Lintner, K. Heat- and UV-stable cosmetic enzymes from deep sea bacteria. Cosmet. Toiletries 2002, 117, 22-30.

25. Lintner, K.; Lamy, F.; Mas-Chamberlin, C.; Mondon, P.; Scocci, S.; Buche, P.; Girard, F. Heat-stable enzymes from Deep Sea Bacteria: A Key Tool for Skin Protection against UV-A induced Free Radicals. IFSCC Mag. 2002, 5, 195-200.

26. Mas-Chamberlin, C.; Mondon, P.; Lamy, F.; Lintner, K. Potential preventive performance. SPC 2006, 79, 38-40.

27. SpecialChem. Sederma's Anti-aging Active Venuceane ${ }^{\mathrm{TM}}$ Shows High Potency against IR-Aging in New Studies. Available online: http://cosmetics.specialchem.com/news/product-news/sedermas-antiagingactive-venuceane-shows-high-potency-against-iraging-in-new-studies (accessed on 22 January 2017).

28. Giacomoni, P.U. Advancement in skin aging: The future cosmeceuticals. Clin. Dermatol. 2008, 26, $364-366$. [CrossRef] [PubMed]

29. Schwarz, T. Mechanisms of UV-induced immunosuppression. Keio J. Med. 2005, 54, 165-171. [CrossRef] [PubMed]

30. Ostad, A. The 12 Best Face Sunscreens. Available online: http:/ /www.redbookmag.com/beauty/anti-aging/ tips/a5027/anti-aging-sunscreen (accessed on 26 October 2016).

31. LIPOTEC Technical Report on Juveleven ${ }^{\circledR}$ Peptide. Available online: https://www.ulprospector.com/ documents /1285110.pdf?bs=2316\&b=236211\&st=20 (accessed on 22 January 2017).

32. Grether-Beck, S.; Marini, A.; Jaenicke, T.; Krutmann, J. Effective photoprotection of human skin against infrared A radiation by topically applied antioxidants: Results from a vehicle controlled, double-blind, randomized study. Photochem. Photobiol. 2015, 91, 248-250. [CrossRef] [PubMed]

(C) 2017 by the author; licensee MDPI, Basel, Switzerland. This article is an open access article distributed under the terms and conditions of the Creative Commons Attribution (CC BY) license (http:/ / creativecommons.org/licenses/by/4.0/). 